\title{
Erratum to: Local models of Shimura varieties and a conjecture of Kottwitz
}

\author{
G. Pappas $\cdot$ X. Zhu
}

Published online: 5 January 2013

(c) Springer-Verlag Berlin Heidelberg 2012

\section{Erratum to: Invent math \\ DOI 10.1007/s00222-012-0442-Z}

Due to a typesetting error the names of the authors in Refs. [51] and [52] were incorrect. The correct references are:

51. Pappas, G., Rapoport, M., Smithling, B.: Local models of Shimura varieties, I. Geometry and combinatorics. Handbook of Moduli (to appear). arXiv:1011.5551

52. Gille, P., Pianzola, A.: Torsors, reductive group schemes and extended affine Lie algebras. Preprint. arXiv:1109.3405 (to appear, Mem. AMS)

The online version of the original article can be found under doi:10.1007/s00222-012-0442-z.

G. Pappas (凶)

Dept. of Mathematics, Michigan State University, E. Lansing, MI 48824, USA

e-mail: pappas@math.msu.edu

X. Zhu

Dept. of Mathematics, Northwestern University, Evanston, IL 60208, USA

e-mail: xinwenz@math.northwestern.edu 